\title{
Core Based Group Communication with Qos Support
}

\author{
Ashok Kumar Bhoi, U.Ghugar Satya Prakash Sahoo, Manas Ranjan Kabat \\ M. Tech Cse, Vssut Burla, Lecturer of Cse, PKACE,Bargarh Lecturer, Dept. Of Cse, Vssut Burla Sr. Lecturer \\ Dept. Cse Vssut Burla
}

\begin{abstract}
The present day is demanding more and more quality of service in broadband group communication to support huge access of internet service and multimedia application. The core based solution is able to full fill this demand a lot. In this paper effort has been put to make it more flexible in comparison to SPAN/COST through a new approach which can be an alternative solution of SPAN/ADJUST for solving the constrained of non singular core solution.
\end{abstract}

Keywords: Multicasting, QoS Routing, Core selection

\section{Introduction}

Data communication for internet uses TCP/IP and UDP protocol for reliability and scalability. It use so many methods like unicast, multicast, anycast and broadcast for audio-video broadcasting, video conferencing and multimedia communication.

It refers to simultaneous delivery of data from multiple sources to multiple receivers of different groups. Group communication leads to multiple paths from a tree structure. In the beginning the source is considered as a root of the multicast tree. A multicast tree is incrementally constructed as members leave and join a multicast group. When an existing member leaves the group, it sends a control message up the tree to prune the branch which has no members attached. The multicasting is used when a lot of information is transmitted to a subset of hosts. Later on a core based tree was introduced in which an intermediate node communicate to remaining down-stream nodes where the core node may also be a source node. The core based multicast tree construct the shortest path between the core and remaining nodes. In section 2, we outline the literature review. In section 3 we introduce SPAN/COST [6] algorithm and cost estimation. In section 4, we evaluate performance of simulation result. We conclude the paper in section 5 .

\section{Related Work}

In routing for group communication usually sender transmits a stream of data to a set of receivers. Storing of destination address was making more and more congested and overloaded in the source node. An alternative node has chosen to treat as core node to share the same overload partially to make the group communication more flexible. That make a core rooted trees for the delivery of their stream. It signify "core" as an intermediate node between sources and receivers including sore node in some cases. The core based models, CBT [1] and PIM [5] uses only single core for their purpose. The motivation behind core based tree is to make scalability and to reduce overhead. A source willing to deliver its stream transmits its data packet to the core from whereon. The ubiquitous use of internet leads to use of multiple cores in a network. The first multi core tree is OCBT [2]. The CBT, PIM, OCBT tried to implement core selection procedure to improve the quality of service. The other core based trees are Greedy, NAÏVE, DDVCA, QCSA, SPAN [6, 7] contributed a lot.

\section{SPAN Framework.}

SPAN [6] is a prominent core selection algorithm which is distributed and asymmetric frame work operates both in singular and non singular solution space. SPAN initially identifies some intermediate nodes having multiple paths between source and receivers and considered as candidate cores. The ultimate cores can be selected based on the optimized cost of the network basing on that core and multipoint path.

\subsection{COST Estimation.}

The cost of a network depends on number of core present in a network. It divides network into number of trees like core to dependent receiver as Rtree and sources to core as Stree. It uses a metric i.e. domination count. Let there is a delay distance path from node $\boldsymbol{i}$ to node $\boldsymbol{j}$ through core $\boldsymbol{c}$ then cost of the tree, $\boldsymbol{C s t}=\operatorname{cost}$ of Rtree + cost of Stree $+\mathbf{D m} . \mathbf{D m}$ is Residual ratio of dominated to un-dominated nodes at the concerned core $\boldsymbol{c}$.

Domination Count:

For a tree $T(c, s)$ rooted at core c serving the source s for all receivers we can express 
Domination Count $(c, s)=\sum_{s^{\prime} \in S}\left|D(c, s) \cap D\left(c, s^{\prime}\right)\right|$

Where $T(c, s)$ serves a source $s^{\prime} \in S$.

Algorithm:

Input: $S, R, C / /$ No. of Source, Receiver and Core

Output: $\boldsymbol{C u} / /$ Ultimate core

1. Find the cost of edges for all receivers in random from \& through cores within a certain range.

2. Find the cost of edges for all sources from all cores.

3. $C u=\Phi$

4. Find $D(c, s)$ for all cores.

5. Find the Cost of the Network, $\boldsymbol{C n} \boldsymbol{w}=\boldsymbol{C s} \boldsymbol{t}$ for all the cores.

6. Find total cost for core $\mathrm{Cl}=\mathrm{Cnw}$

7. Increament $\mathrm{Cl}$ to $\mathrm{C2}$

8. if $\boldsymbol{C n} \boldsymbol{w}$ for $C 1>\boldsymbol{C n} \boldsymbol{w}$ for $C 2$

9. $\mathrm{Cu}=\mathrm{C2}$

10. Else

11. $\mathrm{Cu}=\mathrm{Cl}$

12. Increment the $C 1=C 2+i=1$

\section{Simulation Analysis.}

Simulation has been carried out in MATLAB version 9 in a system having CPU N435 @ 1.33GHz, $1 \mathrm{~GB}$ of RAM. It created a difference from what resulted in SPAN/COST algorithm [6]. In the simulation of SPAN/COST algorithm, input of source nodes, receiver nodes, candidates cores and edges cost required to enter which may be too difficult for a network of node sizes more than hundred or thousand. Here a large number of source nodes, receiver nodes and core nodes can take as an input to get optimized cost. The edges cost are taken randomly giving flexible to run the program in few second. The main advantage is that the cost of each edge can input randomly by the system. Analysis from different combination of sources, receivers with cores are taken and found the following assumption.

1. When number of source increases w.r $t$ number of receiver and core cost of the network increases. [fig-1 \& 5]

2. Cost of the network vary directly to no. of cores and inversely to no. of receivers.[Fig. 2]

3. Keeping number of sources and cores constant, raising the number of receiver decreases the cost of the network.[fig-3]

4. When all source, receiver and core together increases in constant rate the cost of the network decreases.[fig.4]

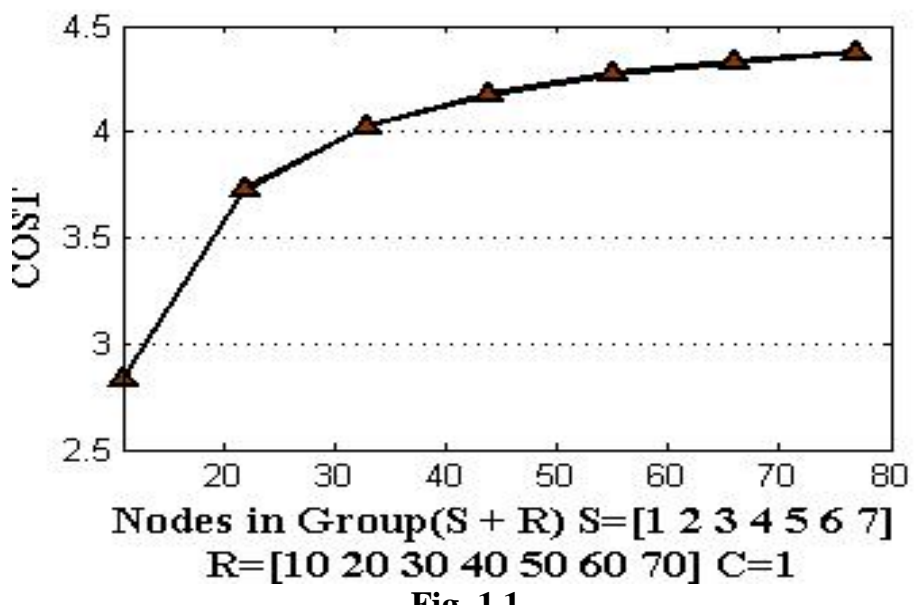

Fig. 1.1 


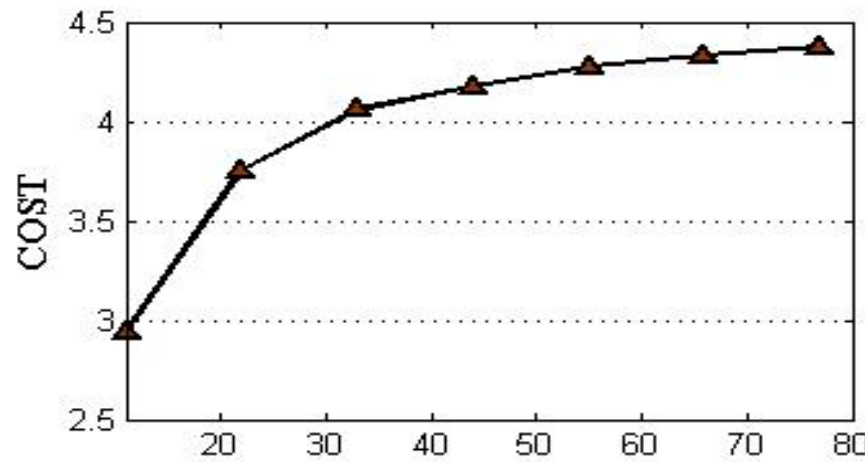

Nodes in Group( $(S+R) S=\left[\begin{array}{lllllll}1 & 2 & 3 & 4 & 5 & 6 & 7\end{array}\right]$ $R=\left[\begin{array}{llllllll}10 & 20 & 30 & 40 & 50 & 60 & 70\end{array}\right] \mathrm{C}=2$

Fig. 1.2

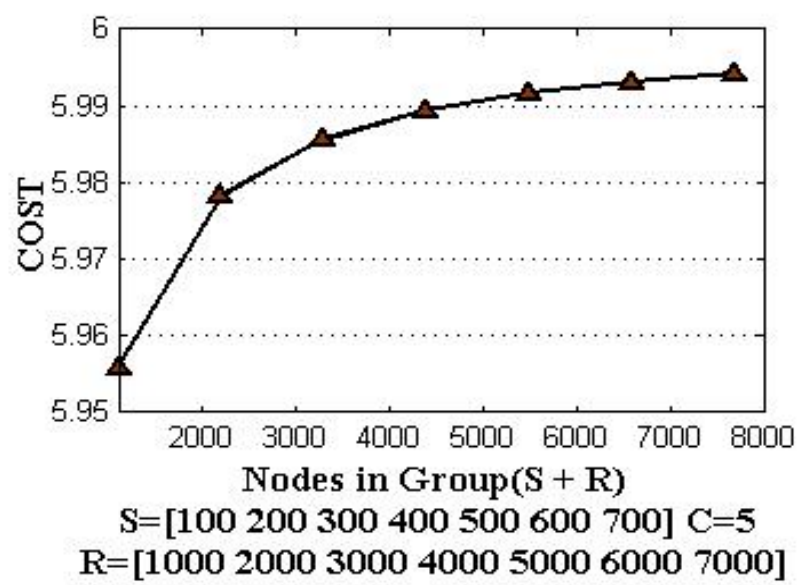

Fig. 1.3

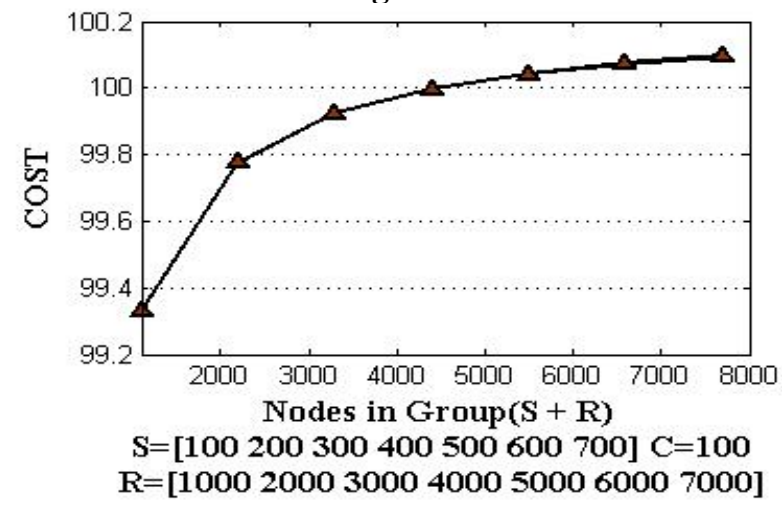

Fig. 1.4

Fig-1 Cost of network vary directly w.r.t. no. of Cores.

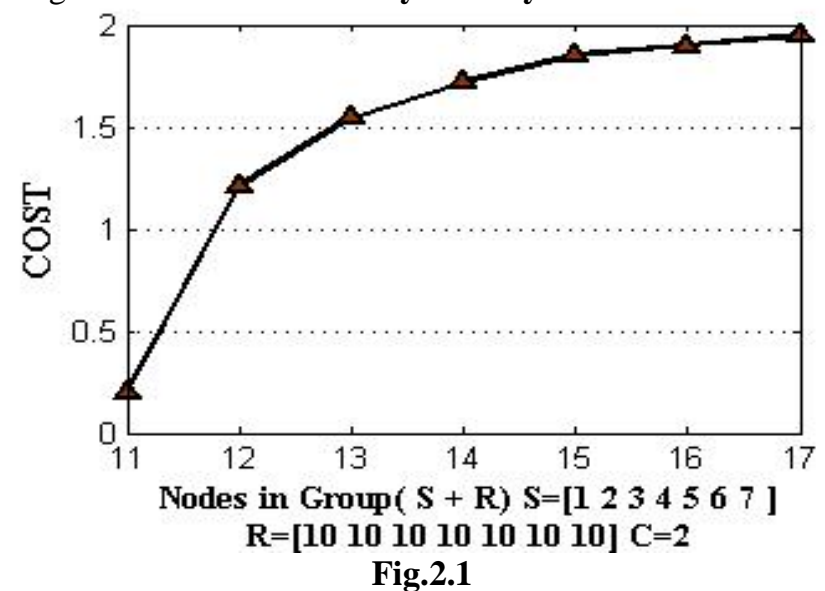



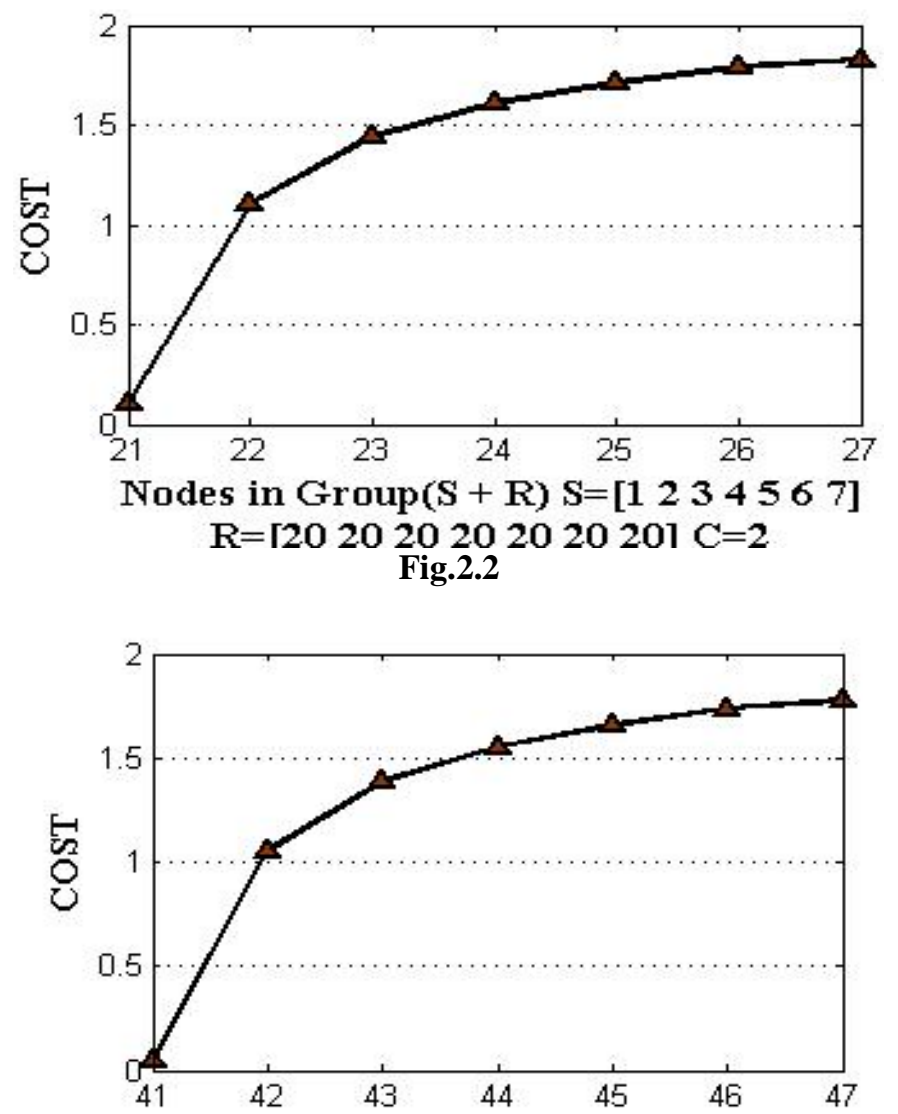

Nodes in Group( $(S+R) S=\left[\begin{array}{lllllll}1 & 2 & 3 & 4 & 5 & 6 & 7\end{array}\right]$

$\mathrm{R}=\left[\begin{array}{ll}404040404040 & 40\end{array}\right] \mathrm{C}=2$

Fig.2.3

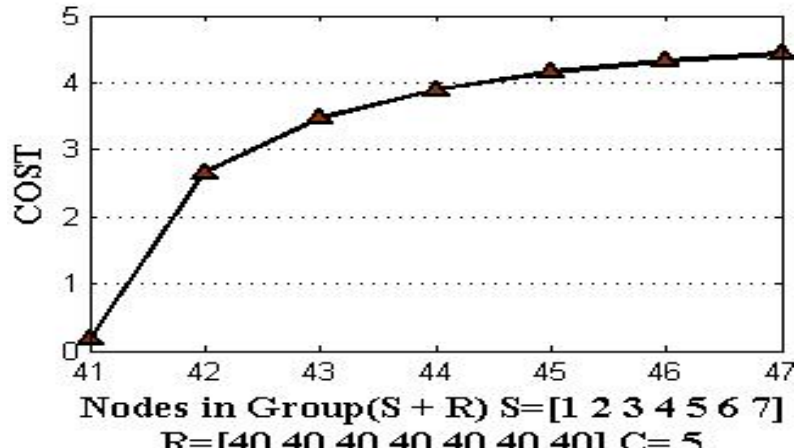

Fig.2.4

Cost Vary directly to no. of cores and inversely to no. of receivers.

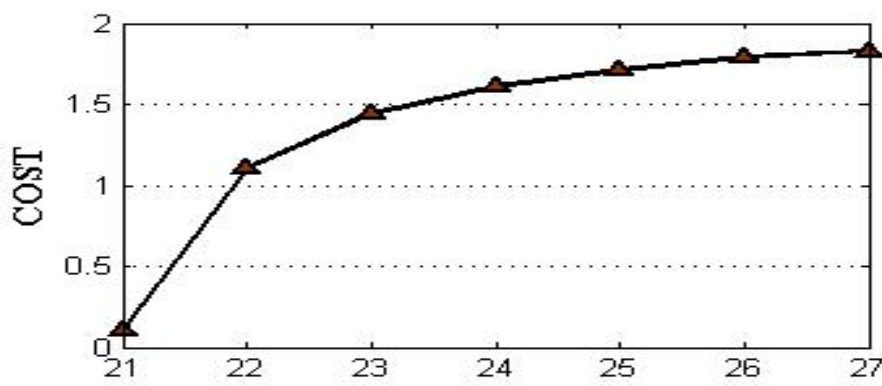

Nodes in Group $(S+R) S=\left[\begin{array}{lllllll}1 & 2 & 3 & 4 & 5 & 6 & 7\end{array}\right]$

$R=\left[\begin{array}{llllllllll}20 & 20 & 20 & 20 & 20 & 20 & 20\end{array}\right] \mathrm{C}=2$

Fig.3.1 


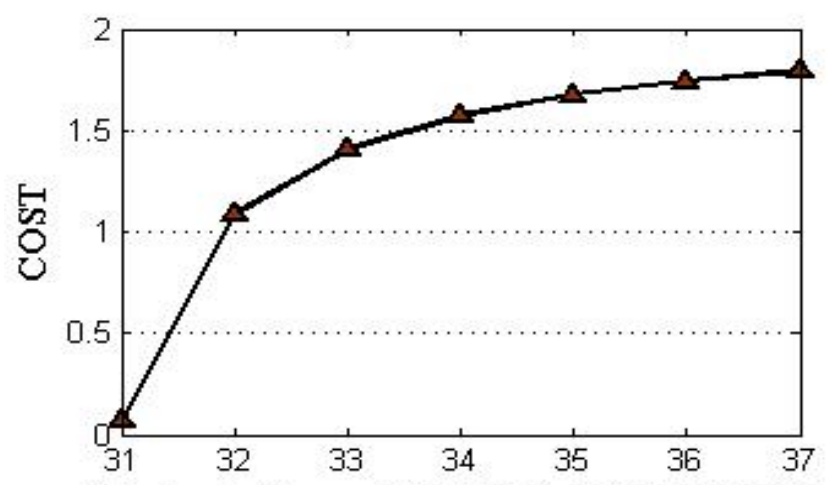

Nodes in Group( $S+R) S=\left[\begin{array}{lllllll}1 & 2 & 3 & 4 & 5 & 6 & 7\end{array}\right]$

$\mathrm{R}=\left[\begin{array}{lllllll}30 & 30 & 30 & 30 & 30 & 30 & 30\end{array}\right] \mathrm{C}=2$

Fig. 3.2

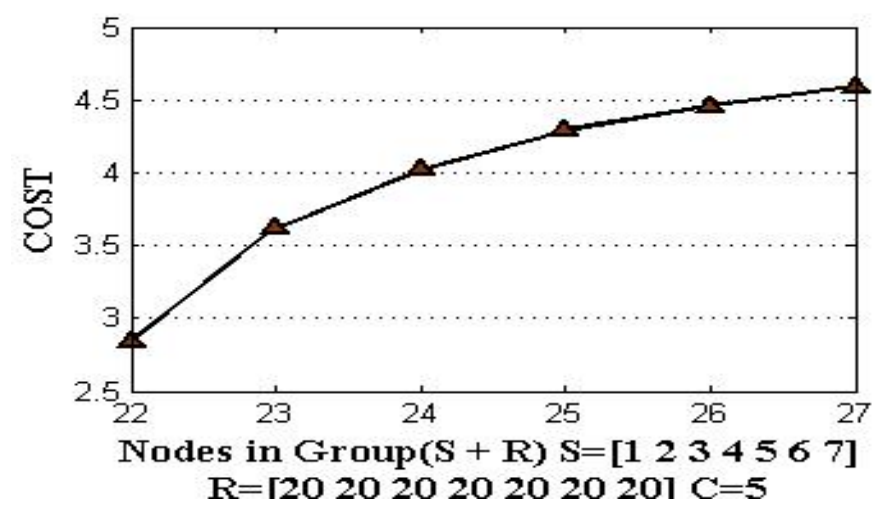

Fig. 3.3

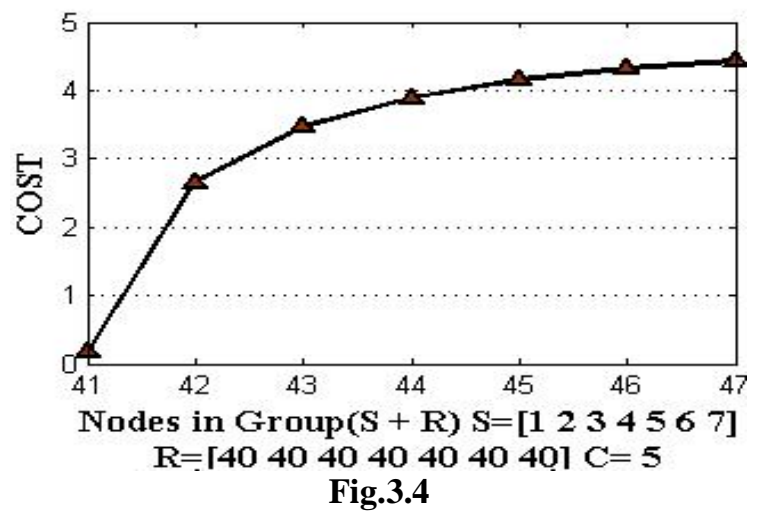

Only Increase in Receiver Decreases the cost.

$$
\text { Fig.3.4 }
$$

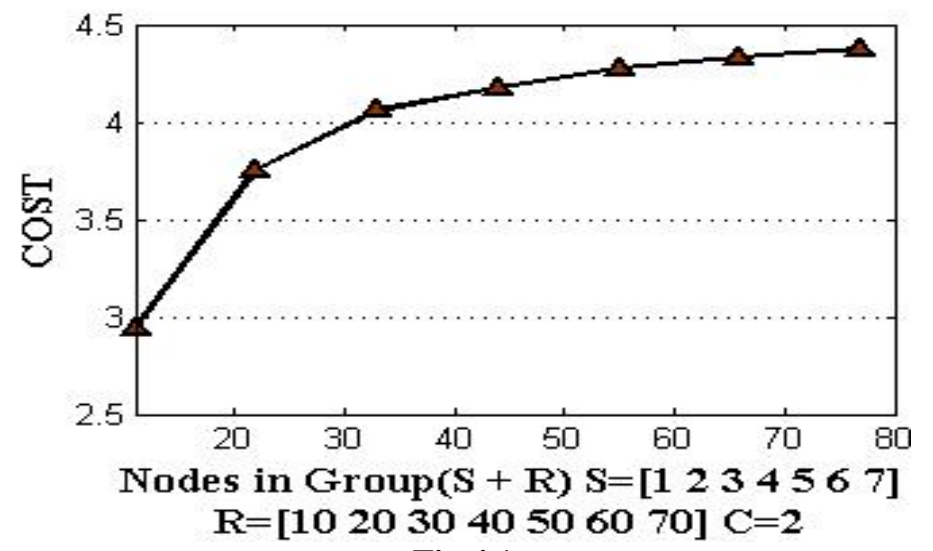

Fig.4.1 


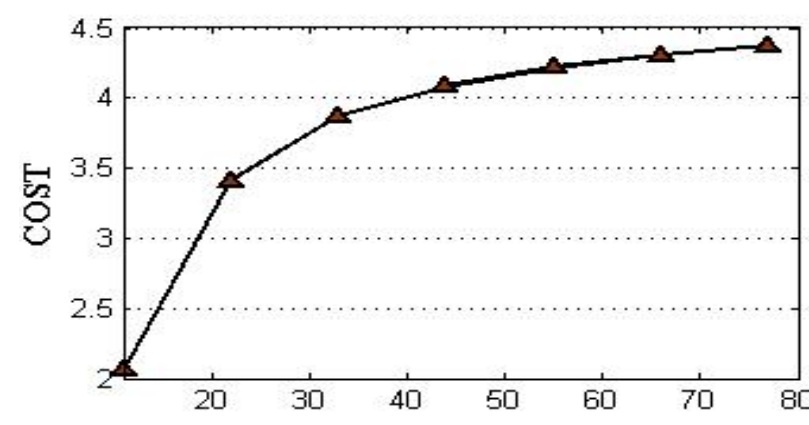

Nodes in Group $(S+R) S=\left[\begin{array}{lllllll}1 & 2 & 3 & 4 & 5 & 6 & 7\end{array}\right]$

$R=\left[\begin{array}{llllllllll}10 & 20 & 30 & 40 & 50 & 60 & 70\end{array}\right] \mathrm{C}=3$

Fig.4.2

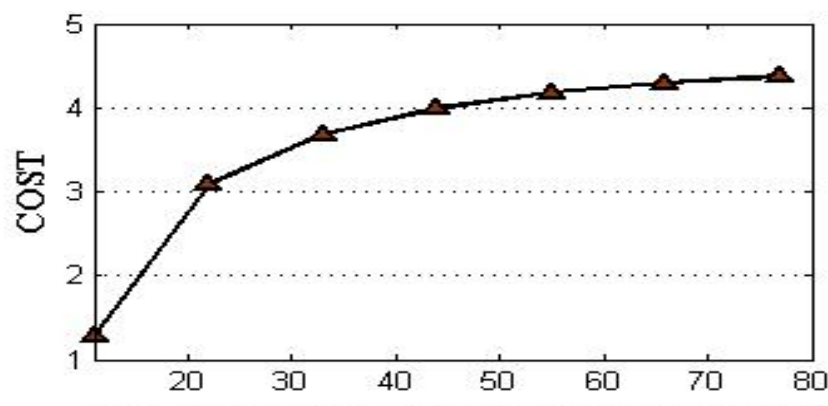

Nodes in Group $(S+R) S=\left[\begin{array}{lllllll}1 & 2 & 3 & 4 & 5 & 6 & 7\end{array}\right]$

$R=[102030405060701 \mathrm{C}=4$

Fig.4.3

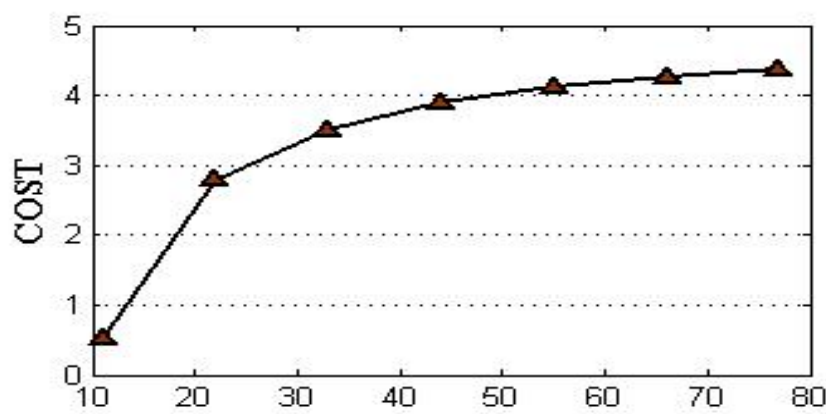

Nodes in Group( $S+R) S=\left[\begin{array}{lllllll}1 & 2 & 3 & 4 & 5 & 6 & 7\end{array}\right]$

$\mathrm{R}=[102030405060701 \mathrm{C}=5$

Fig.4.4

Increase in constant rate of (source, receiver, core ) tends to decrease the cost of the network .

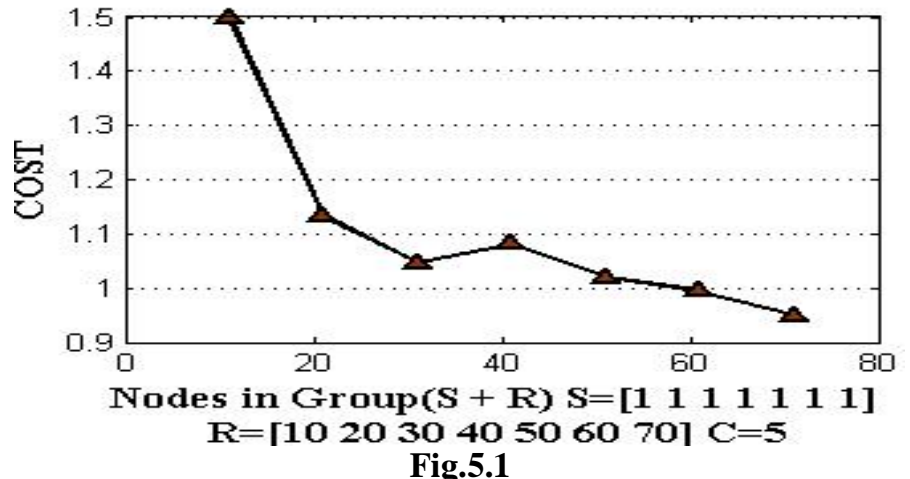




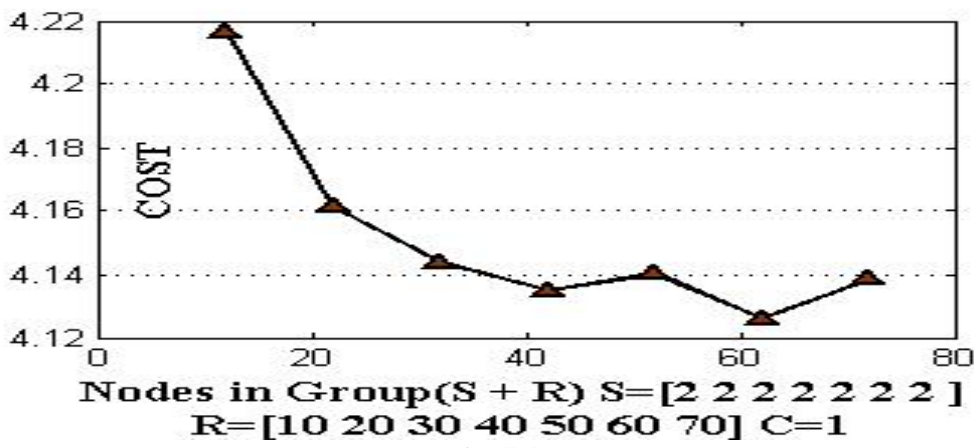

Fig.5.2

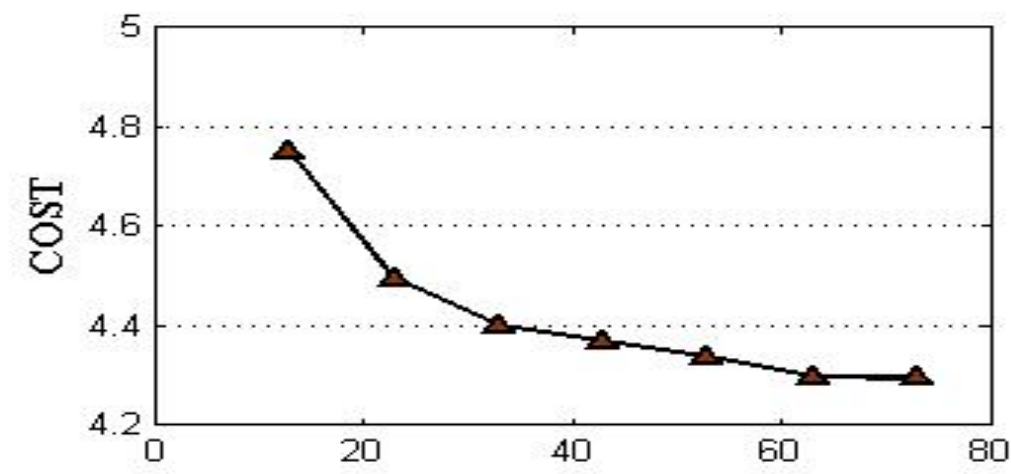

Nodes in Group $(S+R) S=\left[\begin{array}{lllllll}3 & 3 & 3 & 3 & 3 & 3 & 3\end{array}\right]$

Fig.5.3

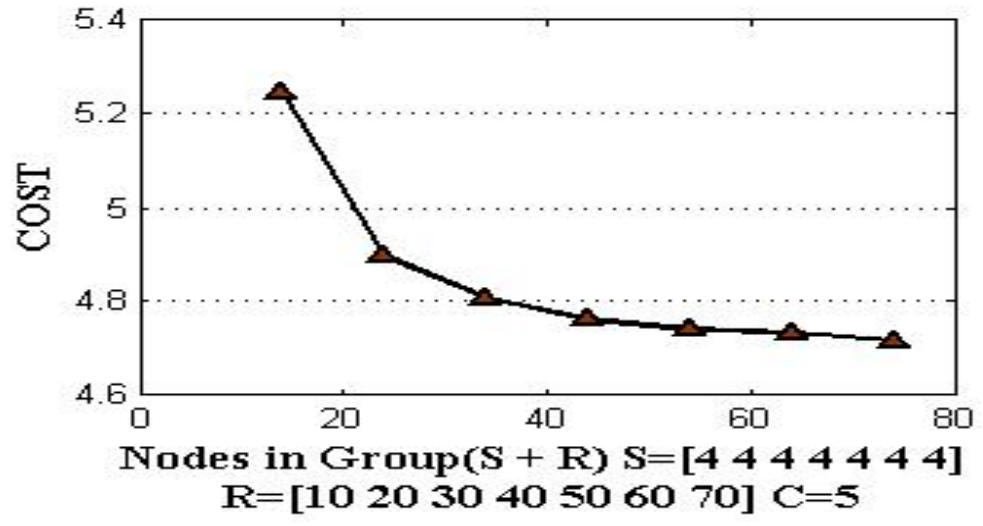

Fig. 5.4

Cost of the network vary directly to increase in number of sources.

\section{Conclusion.}

In this paper it is concluded that ultimate core is not limited to no. of source but instead load of the network. It can calculate the cost of a network having nodes even more than thousand which is difficult in SPAN/COST algorithm. This technique can be improved through increasing the labels of cores in between source and receiver. The dis-advantage of this network is that it may not give the exact value as randomly takes the edge's cost for input give an approximate value. In some special cases of real world constrained it may not be feasible to implement like in sea and forest occupied area.

\section{References:}

[1] Ballardie, Core Based Trees (CBT version 2) Multicast Routing Protocol Specification, RFC 2198, September 1997.

[2] C. Shields, J.J. Garcia-Luna-Acevez, The ordered core based tree, protocol, IEEE INFOCOM (1997).

[3] D. Zappala A. Fabbri, V.Lo, An evaluation of multicast trees with multiple active cores, Journal of Telecommunication Systems,Kluwer, March 2002, pp. 461-479.

[4] A. Ballardie, Core Based Trees (CBT) Multicast Routing Architecture,RFC 2201, September 1997 
[5] H.F. Salama, Multicast routing for real-time communication on high-speed networks, Ph.D., Thesis.

[6] S. Deering, D. Estrin, D. Farinacci, V. Jacobson, Protocol independent multicasting (PIM), dense mode protocol specification, Technical report, IETF-IDMR, 1994.6.

[7] A.Karaman,H.Hassanein.QoS-constrained core selection for group communication. Computer Communications,30(2007),1-13.

[8] Karaman, A., Hassanein, H. S., Extended QoSFrameworkfor Multipoint Communications, to appear,IEEE Symposium on Computers and Communications (ISCC), Alexandria, Egypt, 2004.

[9] W.Putthividhya,W.Tavanapong,M.Tran,J.Wong, Distributed Core Selection with QoS Support, IEEE International Conference on Communications 2004 (ICC'04),June 2004.

[10] B.M.Waxman,Routing of multipoint connections,IEEE Journal on Selected Selected Areas in Communication 6(9)(1988) 1617 1622. 